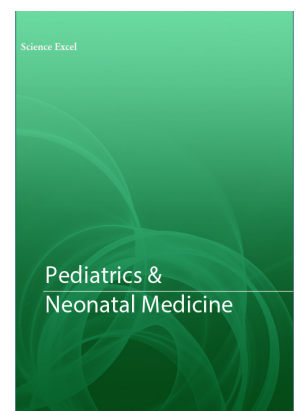

Correspondence

Yuki Asada

Department of Neonatology, Osaka City General Hospital, 2-13-22 Miyakojimahondori, Miyakojima-ku, Osaka 534-0021, Japan

E-mail:mpj_forever_927@yahoo.co.jp

- Received Date: 06 Oct 2020;

- Accepted Date: 15 Oct 2020;

- Publication Date: 17 Oct 2020.
Copyright

(C) 2020 Science Excel. This is an openaccess article distributed under the terms of the Creative Commons Attribution 4.0 International license.

\title{
Meconium obstruction of prematurity diagnosed by exploratory laparotomy on the day of birth
}

\author{
Yuki Asada and Hiroyuki Ichiba \\ Department of Neonatology, Osaka City General Hospital, Osaka, Japan
}

\section{Introduction}

Meconium obstruction of prematurity (MOP) is a distinct clinical condition associated with very low birthweight and generally causes low intestinal obstruction. The etiology is multifactorial and includes immature intestinal function and dysmotility. Potential complications are feeding difficulty, inadequate enteral nutrition and poor growth, bowel perforation, and unnecessary surgical procedures. It is difficult to clinically distinguish MOP from other causes of bowel obstructions such as distal ileal atresia, ileal volvulus, and longsegment Hirschsprung disease. We present a case of MOP diagnosed by exploratory laparotomy on the day of birth.

\section{Case Report}

A 33-year-old gravida 1 para 0 woman, who became pregnant following artificial insemination, was referred to our hospital at 25 weeks' gestation due to the worsening of hypertensive disorders of pregnancy. Fetal hyperechoic bowel was noted by ultrasonography (US) at 23 weeks' gestation, but otherwise she did not have any risk factors. Repeat US showed dilated bowel loops, echogenic bowel, and mural thickening (Figure). A cesarean section was performed at 28 weeks and 1 day of gestation for non-reassuring fetal status. A female baby weighing $679 \mathrm{~g}$ was born and intubated. There were no apparent congenital anomalies. Her abdomen was not bluish, but tortuous loops were visible through the abdominal wall. Plain radiography revealed air-filled distended small bowel loops displaced to the left side. US demonstrated dilated bowel loops that were suggestive of ileal atresia. Gastrografin enemas were attempted, but the contrast agent did not reach the dilated bowel and there was no sign of microcolon. We suspected meconium peritonitis caused by ileal atresia from these findings and fetal US findings. Exploratory laparotomy was performed on the day of birth due to progressively worsening abdominal distention. The most dilated area was $40 \mathrm{~cm}$ proximal to the ileocecum and required an ileostomy. Inspissated meconium was found at $15 \mathrm{~cm}$ proximal to the ileocecum and was removed at the point of ileostomy. No intestinal atresia, perforation or other abnormalities were found. Despite surgical evacuation of the obstructing meconium, irrigation of the proximal end was required post-surgery. Passage of a sufficient volume of stool was confirmed on day 12, and she started to feed from day 20. Hirschsprung's disease was excluded by rectal suction biopsy. The ileostomy was closed on day 75. At this point, her weight was $1730 \mathrm{~g}$. On day 88, full enteral feeding was achieved, and the patient was discharged on day 113 with a body weight of $2440 \mathrm{~g}$.

\section{Discussion}

Bowel obstruction due to meconium frequently occurs in infants with cystic fibrosis (CF) or very low birth weight. The severity ranges from benign meconium plug syndrome to complicated peritonitis that requires ileostomy. Rickham and Boeckman [1] reported the first case of meconium obstruction in a neonate without CF in 1965. In Japan, Kubota et al. [2] proposed the term meconium-related ileus, which encompasses the spectrum of diseases caused by meconium obstruction including MOP. The differential diagnosis of bowel obstruction includes MOP, necrotizing enterocolitis, meconium ileus with CF, Hirschsprung's disease, and intestinal atresia. In the case presented here, dilated bowel loops and echogenic bowel were found on fetal US. No fetal ascites, polyhydramnios or calcification was detected, but the other studies have shown that dilated bowel loops are present in $35 \%$ to $57 \%$ of patients with meconium peritonitis $[3,4]$, and it is difficult 


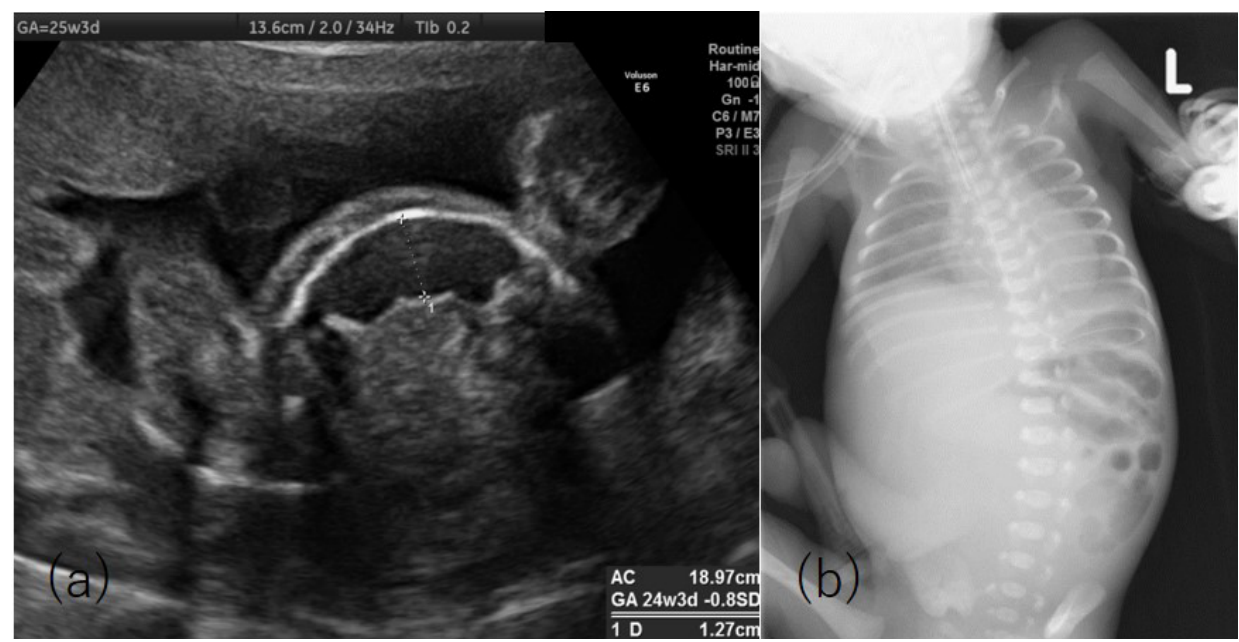

Figure 1: (a) Fetal ultrasound at 25 weeks' gestational age showed a dilated bowel of $12.7 \mathrm{~mm}$ in diameter with mural thickening. (b) A plain radiograph on the day of birth demonstrated a dilated, air-filled small bowel displaced to the left side.

to distinguish MOP from meconium peritonitis or intestinal atresia in the antenatal period. Typically, meconium ileus with CF manifests with dilated bowel loops or echogenic bowel on fetal US [5]. To the best of our knowledge, MOP with dilated bowel loops in the antenatal period has not been described to date. However, Garza-Cox et al. have reported that MOP can be misdiagnosed as intestinal atresia [6]. Microcolon can be found in both MOP and intestinal atresia, but wasn't detected in our case. Likewise, because contrast showed termination of contrast-filled bowel before the distal ileum, diagnosis was not possible. We rather suspected meconium peritonitis with intestinal atresia based on worsening clinical symptoms. In the early stage, MOP is often characterized by the lack of meconium passage, abdominal distention, and bilious vomiting and may be diagnosed based on these symptoms [7]. In our case, a diagnosis was made unexpectedly by exploratory surgery on the day of birth before these symptoms appeared. In conclusion, in-utero diagnosis of MOP by fetal US is challenging, but MOP should be included in the differential diagnosis when antenatal echogenic bowel is detected.

\section{Conflicts of interest}

The authors have no conflicts of interest to declare.

\section{References}

1. Rickham PP, Boeckman CR. Neonatal meconium obstruction in the absence of mucoviscidosis. Am J Surg 1965;109:173-7.

2. Kubota A, Imura $\mathrm{K}$, Yagi $\mathrm{M}$, Kawahara $\mathrm{H}$, Mushiake $\mathrm{S}$, Nakayama M, et al. Functional ileus in neonates. Hirschsprung's disease-allied disorders versus meconium-related ileus. Eur J Pediatr Surg 1999;9:392-5.

3. Shyu MK, Shih JC, Lee CN, Hwa HL, Chow SN, Hsieh FJ. Correlation of prenatal ultrasound and postnatal outcome in meconium peritonitis. Fetal Diagn Ther 2003;18:255-61.

4. Wang CN, Chang SD, Chao AS, Wang TH, Tseng LH, Chang YL. Meconium peritonitis in utero - the value of prenatal diagnosis in determining neonatal outcome. Taiwan J Obstet Gynecol 2008;47:391-6.

5. Garza-Cox S, Keeney SE, Angel CA, Thompson LL, Swischuk LE. Meconium obstruction in the very low birth weight premature infant. Pediatrics 2004;114:285-90.

6. Jerdee T, Newman B, Rubesova E. Meconium in perinatal imaging: associations and clinical significance. Semin Ultrasound CT MR 2015;36:161-77.

7. Dimmitt RA, Moss RL. Meconium diseases in infants with very low birth weight. Semin Pediatr Surg. 2000;9:79-83. 